\title{
Clinical features and antibiotic treatment of septic arthritis and osteomyelitis due to Yersinia enterocolitica
}

\author{
M. CROWE, K. ASHFORD and P. ISPAHANI \\ Department of Microbiology and Public Health Laboratory, Queens Medical Centre, University Hospital, \\ Nottingham NG7 2 UH
}

\begin{abstract}
Yersina enterocolitica bone and joint infections are rare. Over a period of 7 months four patients with deep-seated skeletal infections due to $Y$. enterocolitica were seen at the University Hospital, Nottingham. Sites of infection included the knee (one patient) the hip (one) and the spine (two). None of the patients had major underlying disease or risk factors for developing invasive $\boldsymbol{Y}$. enterocolitica infection. The organisms were sensitive to the second- and third-generation cephalosporins, gentamicin and fluoroquinolones. A literature search covering the period 1970-1994 revealed 20 other cases of skeletal infections due to $\boldsymbol{Y}$. enterocolitica; there was no uniformity in the choice of antimicrobial agent for treating these infections. Oral ciprofloxacin was used as the principal antimicrobial agent in the patients described here and therapeutic success was achieved in three of these patients. Ciprofloxacin should be considered as first line therapy for invasive infections due to $Y$. enterocolitica.
\end{abstract}

\section{Introduction}

Yersinia enterocolitica is recognised increasingly as an enteric pathogen with a wide array of clinical manifestations that vary both with age and health of the host [1-3]. Enterocolitis, the most common presentation, occurs primarily in young children; pseudo-appendicular syndrome, which often mimics acute appendicitis, occurs mainly in older children and young adults; post-infectious manifestations such as reactive arthropathy and erythema nodosum are seen usually in adults.

Less commonly, $Y$. enterocolitica may cause focal infection at extra-intestinal sites with or without bacteraemia. Cases of exudative pharyngitis $[4,5]$, pneumonia, lung abscess, empyema [6-8], liver $[9,10]$, splenic [11], renal [12] or psoas muscle [13] abscesses, endocarditis [14-15], osteomyelitis [16, 17], septic arthritis [18] and meningitis [17] have all been reported. Rarely, in patients with diabetes mellitus, hepatic cirrhosis, blood dyscrasias or in young infants, infection with $Y$. enterocolitica may cause a septicae-

Received 26 Sept. 1995; revised version accepted 18 Feb. 1996.

Corresponding author: Dr M. Crowe. mic illness without an obvious focus of infection $[2,11,19-21]$. Septicaemia caused by transfusion of contaminated blood has also been reported [22, 23].

The great majority of the gastrointestinal infections caused by $Y$. enterocolitica are self-limiting and confined to the gut and do not merit antimicrobial therapy in an immunocompetent host. However, in patients with septicaemia or invasive infection, the mortality can be as high as $50 \% \quad[2,11]$, and antimicrobial therapy is warranted [24]. The organism is usually susceptible in vitro to aminoglycosides, cotrimoxazole, chloramphenicol, tetracycline, the third generation cephalosporins and fluoroquinolones but resistant to penicillin, ampicillin and the first generation cephalosporins. Nevertheless, the drug of choice is yet to be identified $[1,24]$.

Septic arthritis or osteomyelitis caused by $Y$. enterocolitica is extremely rare [25], and experience in the UK of serious infections due to $Y$. enterocolitica and information on the optimal treatment of such infections is limited. This paper reports three patients with proven and one with strongly suspected deep-seated skeletal infection with $Y$. enterocolitica encountered over 7 months and reviews other cases reported in the literature. 


\section{Materials and methods}

Clinical, laboratory and epidemiological findings for each case of $Y$. enterocolitica infection were documented. $Y$. enterocolitica isolates were sent to the Reference Laboratory for Enteric Pathogens (LEP), Central Public Health Laboratory, Colindale, London, for confirmation and typing. Patient sera were also sent to the LEP for serological testing.

Minimal inhibitory concentrations were determined by the agar plate dilution method with a multipoint inoculator as described previously [26].

A search of the English language literature from 1970 to 1994 was conducted via Medline for other reported cases of osteomyelitis and septic arthritis caused by $Y$. enterocolitica. Additional cases were identified through a literature search via Paperchase, a review of cited articles and a subsequent review of the references noted in those articles.

\section{Case reports}

Case 1. An 85-year-old retired monk was hospitalised after a 24-h history of right knee pain and rigors on the day of admission. His past medical history included long-standing osteoarthritis of both knees, cholecystectomy, transurethral prostatectomy, anaemia and proctitis of unknown cause. On admission, his temperature was $38^{\circ} \mathrm{C}$ and his right knee was swollen and tense with a marked reduction in the range of movement. Laboratory findings included: haemoglobin (Hb) $9.9 \mathrm{~g} / \mathrm{dl}$; white blood cell (WBC) count $6.2 \times 10^{9}$ / $\mathrm{L}$; polymorphonuclear leucocyte (PMNL) count of $5.16 \times 10^{9} / \mathrm{L}$; platelet count, $170 \times 10^{9} / \mathrm{L}$; erythrocytic sedimentation rate (ESR), $22 \mathrm{~mm} / \mathrm{h}$. Serum electrolyte concentrations and liver function tests were normal.

A diagnosis of septic arthritis of the right knee was made. Microscopy of a needle aspirate of joint fluid showed numerous neutrophils but no organisms or crystals and treatment was started with intravenous coamoxiclav $(1.2 \mathrm{~g}$ every $8 \mathrm{~h})$. After $48 \mathrm{~h}$, cultures yielded a moderate growth of a 'coliform-type' organism; treatment was changed to cefotaxime $(2 \mathrm{~g}$ every $8 \mathrm{~h}$ ) and gentamicin (120 mg once daily). On day 6, the organism was identified as $Y$. enterocolitica (serotype O9; biotype 3) and treatment was changed to oral ciprofloxacin $750 \mathrm{mg}$ twice daily. The knee was washed out arthroscopically on three occasions and $Y$. enterocolitica was isolated on two of these but before ciprofloxacin was started. A serum sample on day 9 had an agglutination titre of 1280 to the $Y$. enterocolitica isolate.

After 13 days the ciprofloxacin dose was reduced to $500 \mathrm{mg}$ every $12 \mathrm{~h}$. Ciprofloxacin was stopped after 24 days as the WBC count fell to $2.8 \times 10^{9} / \mathrm{L}$ (PMNL count $1.57 \times 10^{9} / \mathrm{L}$ ). He was discharged home and remained well.

Case 2. A 63-year-old housewife was admitted to a local district general hospital with an 8-week history of low back pain, which in the last 3 weeks had increased in intensity and radiated to her left groin and buttock. She gave a history of occasional night sweats. Her past medical history included pulmonary tuberculosis, degenerative disease of the spine, ischaemic heart disease and asthma. Physical examination was unremarkable except for tenderness in the mid-lumbar spinal region and pain on flexion. There was no neurological deficit. Spinal $\mathrm{X}$-rays and magnetic resonance imaging (MRI) suggested vertebral osteomyelitis and an epidural abscess at L3/4 level. She was transferred to the Spinal Unit at the University Hospital, Nottingham for further management. Laboratory findings included: $\mathrm{Hb} 11.3 \mathrm{~g} / \mathrm{dl}$; WBC count $8.5 \times 10^{9} / \mathrm{L}$; PMNL count $5.75 \times 10^{9} / \mathrm{L}$; platelet count $284 \times 10^{9} / \mathrm{L}$ and ESR $>90 \mathrm{~mm} / \mathrm{h}$. Serum electrolytes and liver function tests were normal. A CT-guided biopsy of the L3/4 disk space yielded 4-5 ml of bloodstained fluid; microscopy showed scanty neutrophils and no organisms, but after $48 \mathrm{~h}$ a 'coliform type' organism was isolated. Cefotaxime $(2 \mathrm{~g}$ every $6 \mathrm{~h})$ and gentamicin ( $120 \mathrm{mg}$ once daily) were commenced. Four days later, the organism was identified as $Y$. enterocolitica (serotype 09; biotype 3) and therapy was changed to oral ciprofloxacin ( $750 \mathrm{mg}$ every $12 \mathrm{~h}$ ). She made a rapid and uneventful recovery on medical treatment alone and was discharged on ciprofloxacin ( $750 \mathrm{mg}, 12$ hourly; reduced to $500 \mathrm{mg}, 12$ hourly after 4 weeks). Ciprofloxacin was discontinued after 4 months. A serum sample taken 6 days after admission showed an agglutination titre of 2560 against the $Y$. enterocolitica isolate.

Case 3. A 14-year-old school girl with idiopathic scoliosis who had undergone corrective surgery and stabilisation with instrumentation in November 1992 was admitted in January 1994 with a 3-month history of increasing pain, redness and swelling over the middorsal region of the spinal scar. In November 1993, she had been admitted to the hospital for 3 days with suspected osteomyelitis and started empirically on intravenous cefotaxime and flucloxacillin. However, bone scan results were equivocal, and antibiotics were discontinued. Since then she had been seen by her general practitioner with 'boils' appearing intermittently at different points in the mid and lower back region of the scar, which were being treated locally with Betadine.

On admission her temperature was $38.2^{\circ} \mathrm{C}$ and physical examination revealed four ulcerated areas over the spinal scar. Laboratory findings included: $\mathrm{Hb}$ $10.3 \mathrm{~g} / \mathrm{dl} ; \quad$ WBC count $7.6 \times 10^{9} / \mathrm{L}$; PMNL count $4.77 \times 10^{9} / \mathrm{L} ;$ platelet count $540 \times 10^{9} / \mathrm{L} ; \quad$ ESR $>$ $90 \mathrm{~mm} / 1 \mathrm{~h}$; and C-reactive protein level $45 \mathrm{mg} / \mathrm{L}$. 


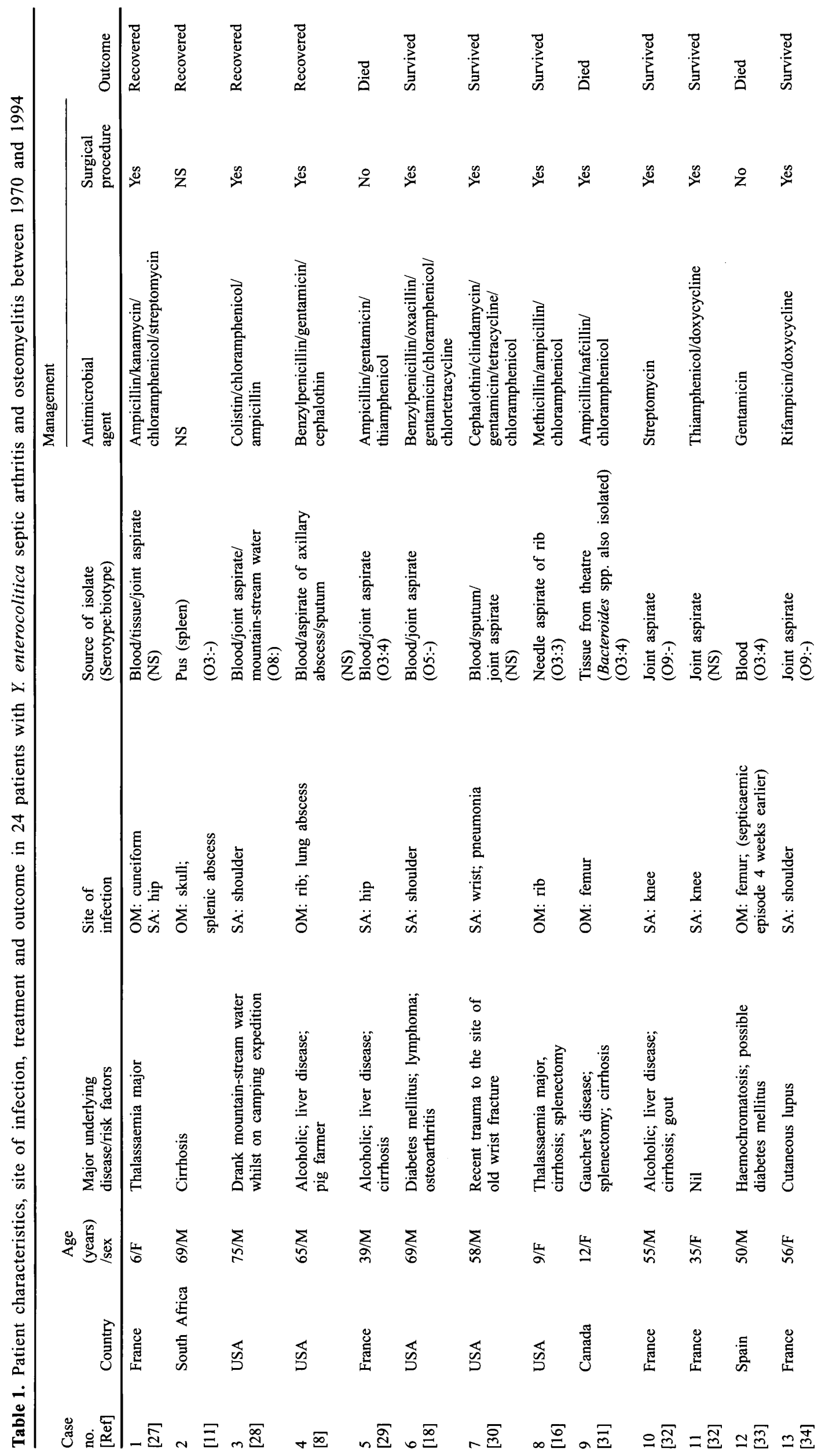




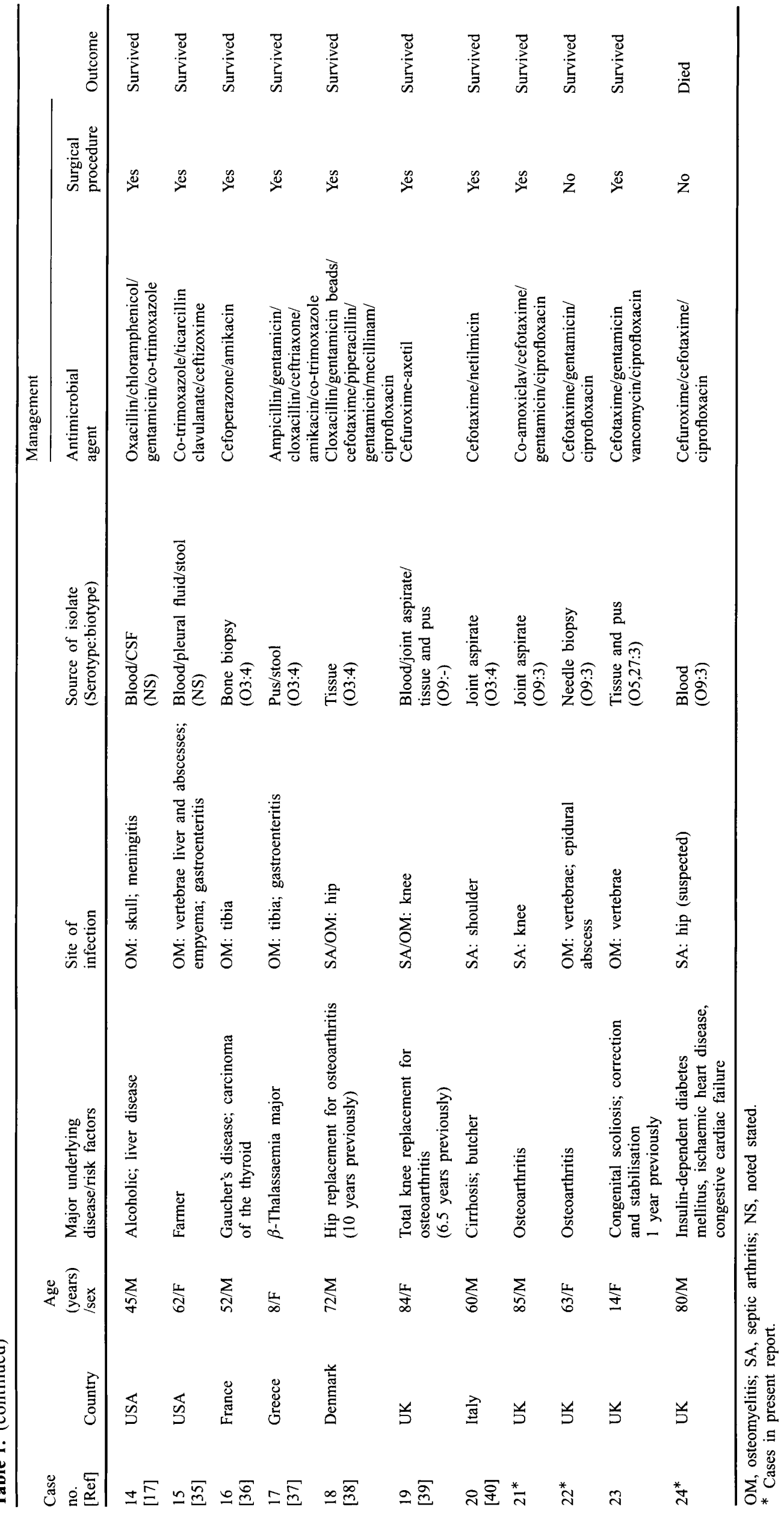


Serum electrolytes and liver function tests were normal.

The following day she underwent exploration of her spinal wound. Granulation tissue and pus was evident along the extent of the metalwork and the pedicle screws were loose. As the spine had fused, all the metalwork was removed and the wound was extensively debrided. Gram-staining of the spinal tissue revealed numerous pus cells but no organisms. The patient was commenced on cefotaxime ( $2 \mathrm{~g}$ every $8 \mathrm{~h}$ ) and vancomycin ( $1 \mathrm{~g} 12$ hourly), as earlier surface swabs taken from the ulcerated areas had yielded coliform bacilli, Staphylococcus aureus and S. epidermidis. After $48 \mathrm{~h} \mathrm{a}$ 'coliform-type' organism was isolated from spinal tissue and gentamicin (120 mg, 8 hourly) was added to the regimen. The organism was identified subsequently as $Y$. enterocolitica (serotype 05.27 ; biotype 3 ); parenteral antibiotics were discontinued and oral ciprofloxacin $500 \mathrm{mg}$ twice daily was commenced. She was discharged 14 days after admission on ciprofloxacin $500 \mathrm{mg}, 12$ hourly for a further 2 weeks followed by ciprofloxacin $250 \mathrm{mg} 12$ hourly for 6 weeks. Serum samples taken during admission were negative for $Y$. enterocolitica antibodies but 6 weeks later a serum sample had an agglutination titre of 320 .

Case 4. An 80-year-old retired security officer was admitted to the hospital following a stroke, leading to a left-sided hemiparesis. His past medical history included insulin-dependent diabetes mellitus, ischaemic heart disease, congestive cardiac failure and bilateral deep vein thrombosis. Initially he made a good recovery but on day 16 became acutely unwell, with fever and pain in his left leg radiating to the hip. A red inflamed area on the medial aspect of the thigh was diagnosed as thrombophlebitis and he was commenced on cefuroxime ( $750 \mathrm{mg}, 8$ hourly) for a presumed chest infection. An ultrasound of the thigh revealed an occluded left great saphenous vein; abdominal ultrasound and echocardiogram were normal.

After $48 \mathrm{~h}$, blood cultures yielded a 'coliform-type' organism and his antimicrobial therapy was changed to cefotaxime ( $1 \mathrm{~g}, 8$ hourly). The organism was identified as $Y$. enterocolitica (serotype O9; biotype 3 ) and treatment was changed to oral ciprofloxacin (500 mg every $12 \mathrm{~h}$ ). However, the patient continued to deteriorate; he remained febrile and complained of increasing pain in the left hip. A clinical diagnosis of septic arthritis was made. In view of his other medical problems, it was considered inappropriate to investigate the hip joint further. He died 10 days after he first become febrile.

\section{Literature review and discussion}

The salient features of the cases described here and 20 previously reported cases of skeletal infection due to $Y$. enterocolitica $[8,11,16-18,27-40]$ are summarised in Table 1.

\section{Epidemiology}

Of 20 reported cases of skeletal infections due to $Y$. enterocolitica, only one was from the UK, the remaining patients were from France (six cases), USA (seven cases) and one each from South Africa, Canada, Spain, Greece, Denmark and Italy. There were 12 males and eight females. The mean age of the patients was 49 years (range 6-84 years). The four children were all females (Table 1). The duration of illness in the reported cases was variable, lasting from 1 day to many weeks, similar to our experience with the four patients in the current series.

Y. enterocolitica has been isolated from soil, vegetables, water, wild and domestic animals and animal products including raw milk, whipped cream, icecream, beef, lamb and poultry. Serotypes most commonly isolated from human infections have also been isolated from the upper respiratory tract and faeces of pigs [1,2]. In man, the most common portal of entry for $Y$. enterocolitica is the gastrointestinal tract following ingestion of contaminated food, water or milk. Consumption of contaminated raw or inadequately cooked pork probably accounts for the majority of sporadic human infections [41, 42], although direct oral or faecal-oral transmission from dogs, cats and pigs to man have been reported [1,2]. Indeed there is an increased risk of $Y$. enterocolitica infections in those likely to come in contact with infected animals or their products, e.g., abattoir workers [43]. The occupation of the three adult patients (Cases 4, 15 and 20, Table 1) in those reviewed is, therefore, of interest.

No definite source of infection was identified in any of the four patients reported here. Patients 1 and 2 gave no history of recent gastrointestinal symptoms, intake of high risk foods, or contact with animals. Patient 3 received a blood transfusion in November 1992, at the time of her last spinal surgery, but it is unlikely that she was infected through this route as her symptoms did not start until September 1993. However, she had visited a farm with other school children in July 1993. Patient 4 had been an in-patient in a general medical ward for 16 days before he became acutely unwell with fever for the first time. However, he had an episode of diarrhoeal illness several weeks before admission.

\section{Pathogenesis}

Besides geographic differences in the frequency and distribution of $Y$. enterocolitica infection, there are differences in virulence among $Y$. enterocolitica strains. Isolates of serotypes $03, \mathrm{O} 9$ and $\mathrm{O} 8$ are the most frequent causes of sporadic human disease worldwide 
[1]. Serotypes of 14 of the 20 isolates of $Y$. enterocolitica from skeletal infections were documented in the reports reviewed; nine belonged to serotype $\mathrm{O} 3$ and three to serotype O9. Three of the four isolates in the present series belonged to serotype O9; the serotype 05.27 isolate from patient 3 has not been reported as a cause of skeletal infection, but is known to cause disease in man $[41,42]$.

Iron is an essential growth factor for the multiplication of most bacteria, which obtain iron by releasing highaffinity chelators called siderophores. $Y$. enterocolitica is unusual amongst micro-organisms in being unable to produce siderophores, but has appropriate receptors and can use siderophores from other sources [44]. Invasive infections due to $Y$. enterocolitica have been reported most frequently in patients with conditions associated with iron overload, including thalassaemia and haemochromatosis, and immunocompromised patients [2, 10-12]. Amongst reported cases of skeletal infection due to $Y$. enterocolitica, three of four children had thalassaemia major, and the fourth child had Gaucher's disease, cirrhosis and had undergone splenectomy (Table 1); seven of the 16 adults had hepatic dysfunction (cirrhosis, five patients; alcoholic liver disease, one; Gaucher's disease, one). Rabson et al. described 13 patients with extra-intestinal infections due to $Y$. enterocolitica, nine of whom had cirrhosis [11]. It has been suggested that investigation for diseases associated with iron overload, particularly haemochromatosis, be conducted on patients with invasive infection due to $Y$. enterocolitica $[10,45]$. However, Hoogkamp-Korstange, in a prospective study of 189 patients with $Y$. enterocolitica infections in The Netherlands, recorded 39 patients (mainly adults) with extra-intestinal infection with no predisposing factors [46]. None of the four patients in the current series and five patients reported earlier (Cases 3, 7, 11, 18 and 19) had underlying conditions associated with invasive $Y$. enterocolitica infection. Serum ferritin levels measured during the follow-up period in the three living patients in the current series were found to be within normal limits.

\section{Site of infection and diagnosis}

All the reported cases of $Y$. enterocolitica skeletal infection were diagnosed by isolating the organism either directly from the site of infection or blood cultures, or both. In the 16 patients in whom blood cultures were documented as being obtained, 10 were positive; in our series blood cultures were positive in only one of the four patients.

Of 10 patients reported with $Y$. enterocolitica septic arthritis, two developed infection in prosthetic joints, and four involved the shoulder, an unusual site of septic arthritis. Nine patients had osteomyelitis, and one patient had both osteomyelitis and septic arthritis. Haematogenous osteomyelitis of the long bones is rare in adults [25]. Cases 12 and 16 (Table 1), both of whom had major underlying diseases, developed osteomyelitis of the femur and tibia, respectively. There has been only one previous report of vertebral osteomyelitis caused by $Y$. enterocolitica (Case 15). We encountered two such patients, one with a complicating epidural abscess.

\section{Treatment}

$Y$. enterocolitica isolates frequently produce $\beta$-lactamases and are often resistant to penicillin, ampicillin and the first generation cephalosporins $[47,48]$. The WHO recommendations for antimicrobial chemotherapy for $Y$. enterocolitica infections include tetracycline, chloramphenicol, gentamicin and cotrimoxazole [49]. More recently, the third generation cephalosporins and the fluoroquinolones, which have excellent in-vitro activity against $Y$. enterocolitica, have been considered as alternatives for treating $Y$. enterocolitica infections $[46,48]$. The four $Y$. enterocolitica isolates from the present report were susceptible to cefuroxime $(\mathrm{MIC} \leqslant 4 \mathrm{mg} / \mathrm{L})$, cefotaxime $(\leqslant 0.12)$, ceftazidime (0.25), imipenem (0.25), gentamicin (1.0) and ciprofloxacin (0.03). A recent review of 53 documented cases of $Y$. enterocolitica septicaemia between 1985 and 1991 reported a mortality rate of only $7.5 \%$, compared to $50 \%$ mortality pre- 1971 ; this success was attributed partly to the use of these newer agents [50]. However, the authors noted that patients who received third generation cephalosporins had a lower cure rate $(85 \%)$ and remained febrile for longer (mean 5.8 days) than patients who received fluoroquinolones (cure rate, 100\%; fever response, mean 2 days). Individual case reports of failure of cefotaxime treatment in $Y$. enterocolitica invasive infections [51] and a poor response to treatment with other cephalosporins (e.g., cefuroxime, ceftazidime and cefoperazone), despite in-vitro activity, have also been noted by others $[46,50]$. Furthermore, in a mouse model of systemic infection with $Y$. enterocolitica, a regimen of high dose cefotaxime or imipenem was found to be inferior to gentamicin or doxycycline [52]. Lemaitre et al. [53], in the same mouse model, showed greater efficacy of fluoroquinolones compared to $\beta$-lactam antibiotics in the treatment of murine $Y$. pseudotuberculosis infection and cautioned against third generation cephalosporins for the therapy of human yersiniosis. The reasons for the discrepancy between in-vitro and in-vivo efficacy of the $\beta$-lactam antibiotics, including the third generation cephalosporins, in the treatment of $Y$. enterocolitica infections are not clear. A wide variety of antimicrobial agents was used in the treatment of skeletal infections with $Y$. enterocolitica (Table 1), including aminoglycosides (12 cases), chloramphenicol (nine), tetracycline (four) and co-trimoxazole (three). A second or a third generation cephalosporin was used at some stage in six of the 20 patients reported. Cefotaxime treatment was considered to have failed clinically and bacteriologically in two cases; case 18 
had received cefotaxime for 8 days following removal of the prosthesis yet $Y$. enterocolitica was still isolated from drainage sites; the organism was re-isolated from the shoulder joint aspirate of case 20 despite 16 days of cefotaxime (1g every $8 \mathrm{~h}$ ). However, second and third generation cephalosporins either alone or in combination were successful in Cases 15, 16, 17 and 19.

Ciprofloxacin was used in only one patient (Case 18) after cefotaxime had failed and the patient had also received courses of piperacillin plus gentamicin and mecillinam. Fluoroquinolones have been used successfully to treat $Y$. enterocolitica septicaemia, liver abscess and pericarditis [45, 54]. Explanations offered for the greater success of this group of agents over the recent $\beta$-lactam antibiotics include its excellent invitro activity, superior tissue penetration and intracellular activity.

In the series reported here, oral ciprofloxacin was used as a single agent once the organism was identified as $Y$. enterocolitica. We are not aware of any other report of deep-seated skeletal infection due to $Y$. enterocolitica being treated successfully with oral ciprofloxacin. Ciprofloxacin should be considered as the first line agent for treating invasive infections due to $Y$. enterocolitica. However, close and frequent monitoring of full blood count is warranted when ciprofloxacin is used for a prolonged period, as it may result in leucopenia [55].

We thank the four consultants (Dr N.G. Smith, Miss H. Prince, Mr J. Webb and Dr S.P. Allison) for permission to study patients under their care, colleagues in the Department of Microbiology for their technical expertise, friends for translating foreign language articles and Mrs Susan Brailsford for typing the manuscript. Finally, we are grateful to Mr T. Cheasty of the Laboratory of Enteric Pathogens, Central Public Health Laboratory, Colindale for advice, and to his colleages for $Y$. enterocolitica typing and serology.

\section{References}

1. Cover TL, Aber RC. Yersinia enterocolitica. $N$ Engl $J$ Med 1989; 321: 16-24.

2. Mollaret HH. L'infection humaine a Yersinia enterocolitica en 1970, a la lumiere de 642 cas recents: aspects cliniques et perspectives epidemiologiques. Pathol Biol (Paris) 1971; 19: 189-205.

3. Johnson RH. Yersinia infections. Curr Opin Infect Dis 1992; 5: 654-658.

4. Tacket CO, Davis BR, Carter GR, Randolph JF, Cohen ML. Yersinia enterocolitica pharyngitis. Ann Intern Med 1983; 99: $40-42$.

5. Rose FB, Camp CJ, Antes EJ. Family outbreak of fatal Yersinia enterocolitica pharyngitis. Am J Med 1987; 82 (3, special no.): 636-637.

6. Clarridge J, Roberts C, Peters J, Musher D. Sepsis and empyema caused by Yersinia enterocolitica. J Clin Microbiol 1983; 17: 936-938.

7. Kane DR, Reuman PD. Yersinia enterocolitica causing pneumonia and empyema in a child and a review of the literature. Paed Infect Dis $J$ 1992; 11: 591-593.

8. Sebes JI, Mabry EH, Rabinowitz JG. Lung abscess and osteomyelitis of rib due to Yersinia enterocolitica. Chest 1976; 69: $546-548$.

9. Nemoto H, Murabayashi K, Kawamura Y et al. Multiple liver abscesses secondary to Yersinia enterocolitica. Intern Med 1992: 31: 1125-1127.

10. Vadillo M, Corbella X, Pac V, Fernandez-Viladrich P, Pujol R. Multiple liver abscesses due to Yersinia enterocolitica discloses primary hemochromatosis: three case reports and review. Clin Infect Dis 1994; 18: 938-941.

11. Rabson AR, Hallett AF, Koornhof HJ. Generalized Yersinia enterocolitica infection. J Infect Dis 1975; 131: 447-451.

12. Shibuya H, Ando M, Kuroda K, Naoe S, Maruyama T. Fatal Yersinia enterocolitica septicemia complicated by unique hemosiderosis. A case report. Acta Pathol Jpn 1988; 38: 1461-1472.

13. Kahn FW, Glasser JE, Agger WA. Psoas muscle abscess due to Yersinia enterocolitica. Am J Med 1984; 76: 947-949.

14. Appelbaum JS, Wilding G, Morse LJ. Yersinia enterocolitica endocarditis. Arch Intern Med 1983; 143: 2150-2151.

15. Giamarellou H, Antoniadou A, Kanavos K, Papaioannou C, Kanatakis S, Papadaki K. Yersinia enterocolitica endocarditis: case report and literature review. Eur J Clin Microbiol Infect Dis 1995; 14: 126-130.

16. Thirumoorthi MC, Dajani AS. Yersinia enterocolitica osteomyelitis in a child. Am $J$ Dis Child 1978; 132: 578-580.

17. Casey MF, Gilligan PH, Smiley ML. Yersinia enterocolitica meningitis and osteomyelitis: a case report. Diagn Microbiol Infect Dis 1987; 8: 47-50.

18. Spira TJ, Kabins SA. Yersinia enterocolitica septicemia with septic arthritis. Arch Intern Med 1976; 136: 1305-1308.

19. Chessum B, Frengley JD, Fleck DG, Mair NS. Case of septicaemia due to Yersinia enterocolitica. BMJ 1971; 3: 466.

20. Cherchi GB, Pacifico L, Cossellu S et al. Prospective study of Yersinia enterocolitica infection in thalassemic patients. Pediatr Infect Dis 1995; 14: 579-584.

21. Challapalli M, Cunnigham DG. Yersinia enterocolitica septicemia in infants younger than three months of age. Pediatr Infect Dis $J$ 1993; 12: 168-169.

22. Jacobs J, Jamaer D, Vandeven J, Wouters M, Vermylen C, Vandepitte J. Yersinia enterocolitica in donor blood: a case report and review. $J$ Clin Microbiol 1989; 27: 1119-1121.

23. Hastings JGM, Batta K, Goure-vitch D et al. Fatal transfusion reaction due to Yersinia enterocolitica. J Hosp Infect 1994; 27: 75-79.

24. Butler T. Yersinia species (including plague). In: Mandell GL, Bennett JE, Dolin R (eds) Mandell, Douglas and Bennett's Principles and practice of infectious diseases, 4th edn, vol 2. New York, Churchill Livingstone. 1995: 2070-2078.

25. Crenshaw AH (ed). Campbell's operative orthopaedics, 8 th edn, vol 1. St Louis, Missouri, Mosby Year Book Inc. 1992: 119-191.

26. Working Party of the British Society for Antimicrobial Chemotherapy. A guide to sensitivity testing. $J$ Antimicrob Chemother 1991; 27 Suppl D: 1-50.

27. Blum D, Viart P, Dachy A. Septicemie a Yersinia enterocolitica chez deux enfants atteints de thalassemie majeure. Arch $\mathrm{Fr}$ Pediatr 1970; 27: 445.

28. Keet EE. Yersinia enterocolitica septicemia. Source of infection and incubation period identified. NY State J Med 1974; 74: $2226-2230$.

29. Sirot J, Morin B, Briat JP et al. Deux cas de suppuration profonde a Yersinia enterocolitica. Med Mal Inf 1976; 6: 329334.

30. Taylor BG, Zafarzai MZ, Humphreys DW, Manfredi F. Nodular pulmonary infiltrates and septic arthritis associated with Yersinia enterocolitica bacteremia. Am Rev Respir Dis 1977; 116: $525-529$.

31. Lafleur L, Hammerberg O, Delage G, Pai CH. Yersinia enterocolitica infection in children: 4 years experience in the Montreal urban community. Contrib Microbiol Immunol 1979; 5: 298-303.

32. Ziegler G, Euller L, Dellamonica P, Etesse H. Deux cas d'arthrite a Yersinia enterocolitica avec mise en evidence du germe intra-articulaire. Rev Rhum 1981; 48: 118-120.

33. Molins A, Fernandez-Guerrero ML, Torres A, Jimenez MP, Soriano F, Abad J. Bacteriemia de brecha y osteomielitis causada por Yersinia enterocolitica. Rev Clin Esp 1982; 165: 363-364.

34. Treves R, Jacob P, Arnaud M, et al. Arthrite a Yersinia enterocolitica avec presence de germe intra-articulaire. Rev Rhum 1985; 52: 503.

35. Sinnott JT, Multhopp H, Leo J, Rechtine G. Yersinia 
enterocolitica causing spinal osteomyelitis and empyema in a nonimmunocompromised host. South Med J 1989; 82: 399400.

36. Fisch A, Prazuck T, Haroche G, Dublanchet A, Lafaix C Hematogeneous osteitis due to Yersinia enterocolitica. $J$ Infect Dis 1989; 160: 554

37. Kansouzidou A, Danielides VD. Yersinia enterocolitica osteomyelitis in a child with B-thalassemia. Clin Microbiol News 1990; 12: 23-24.

38. Hougaard K, Sogaard P. Yersinia enterocolitica coxitis after hip replacement. A case report. Acta Orthop Scand 1990; 61: 364366.

39. Oni JA, Kangesu T. Yersinia enterocolitica infection of a prosthetic knee joint. Br J Clin Pract 1991; 45: 225.

40. Tiddia F, Cherchi GB, Pacifico L, Chiesa C. Yersinia enterocolitica causing suppurative arthritis of the shoulder. $J$ Clin Pathol 1994; 47: 760-761.

41. Tauxe RV, Vandepitte J, Wauters G et al. Yersinia enterocolitica infections and pork: the missing link. Lancet 1987; 1: 1129-1132.

42. Lee LA, Taylor J, Carter GP, Quinn B, Farmer JJ, Tauxe RV and the Yersinia enterocolitica Collaborative Study Group. Yersinia enterocolitica $0: 3$ : an emerging cause of pediatric gastroenteritis in the United States. J Infect Dis 1991; 163: $660-663$.

43. Merilahti-Palo R, Lahesmaa R, Gransfors $\mathrm{K}$, GripenbergLerche C, Toivanen P. Risk of Yersinia infections among butchers. Scand J Infect Dis 1991; 23: 55-61.

44. Robins-Browne RM, Prpic JK. Effects of iron and desferrioxamine on infections with Yersinia enterocolitica. Infect Immun 1985; 47: 774-779.

45. Collazos J, Guerra E, Fernandez A, Mayo J, Martinez E. Miliary liver abscesses and skin infection due to Yersinia enterocolitica in a patient with unsuspected hemochromatosis Clin Infect Dis 1995; 21: 223-224.

46. Hoogkamp-Korstanje JAA. Antibiotics in Yersinia enterocolitica infections. J Antimicrob Chemother 1987; 20: 123-131.

47. Cornelis G, Abraham EP. Beta-lactamases from Yersinia enterocolitica. J Gen Microbiol 1975; 87: 273-284.

48. Hornstein MJ, Jupeau AM, Scavizzi MR, Philippon AM, Grimont PAD. In vitro susceptibilities of 126 clinical isolates of Yersinia enterocolitica to 21 beta-lactam antibiotics. Antimicrob Agents Chemother 1985; 27: 806-811.

49. World Health Organization. Yersiniosis. EURO reports and studies No. 60. Reports on a WHO meeting 1981. WHO regional office for Europe, Copenhagen, 1983

50. Gayraud M. Scavizzi MR, Mollaret HH, Guillevin L. Hornstein MJ. Antibiotic treatment of Yersinia enterocolitica septicemia: a retrospective review of 43 cases. Clin Infect Dis 1993; 17 $405-410$.

51. Noble RC. Failure of cefotaxime in the treatment of Yersinia enterocolitica sepsis despite in vitro susceptiblity. Curr Ther Res 1989; 46: 692-694.

52. Scavizzi MR, Alonso JM, Philippon AM, Jupeau-Vessieres AM, Guiyoule A. Failure of newer beta-lactam antibiotics for murine Yersinia enterocolitica infection. Antimicrob Agents Chemother 1987; 31: 523-526.

53. Lemaitre BC, Mazigh DA, Scavizzi MR. Failure of $\beta$-lactam antibiotics and marked efficacy of fluoroquinolones in treatment of murine Yersinia pseudotuberculosis infection. Antimicrob Agents Chemother 1991: 35: 1785-1790.

54. Lecomte F, Eustache M, Lemeland J-F, Oksenhendler G, Humbert G. Purulent pericarditis due to Yersinia enterocolitica. $J$ Infect Dis 1989; 159: 363.

55. Choo PW, Gantz NM. Reversible leukopenia related to ciprofloxacin therapy. South Med J 1990; 83: 597-598. 